\title{
Role of thrombophilia in splanchnic venous thrombosis in acute pancreatitis
}

\author{
Sobur Uddin Ahmeda, Surinder S. Ranaa , Jasmina Ahluwalia ${ }^{b}$, Neelam Varma ${ }^{b}$, Ravi Sharma ${ }^{a}$, Rajesh \\ Guptac, Mandeep Kang ${ }^{\text {d }}$
}

Post Graduate Institute of Medical Education and Research (PGIMER), Chandigarh, India

\section{Abstract}

\begin{abstract}
Background Splanchnic venous thrombosis (SVT) is a common vascular complication of acute pancreatitis (AP). We conducted this study to prospectively investigate the frequency, risk factors, and extent of SVT in patients with AP and to evaluate the role of thrombophilia in its causation.
\end{abstract}

Methods Patients with AP presenting between January 2015 and June 2016 were prospectively evaluated with contrast-enhanced computed tomography (CT) for the presence of SVT. These patients underwent a detailed analysis of coagulation parameters.

Results We evaluated 96 patients with AP (73 male, mean age $31.85 \pm 13.34$ years), of whom $26(27.1 \%)$ had SVT. Splenic vein, portal vein, and superior mesenteric vein involvement were seen in 22 (84.6\%), 11 (42.3\%), and 4 (15.3\%) patients, respectively. Necrotizing pancreatitis, CT severity index (CTSI) $>6$ and modified CTSI $>6$ were significantly more frequent in patients with SVT than in those without $(96.2 \%$ vs. $78.6 \%, 76.9 \%$ vs. $47.1 \%$, and $92.3 \%$ vs. $67.1 \%$, respectively). Proteins C and S, and antithrombin III deficiency were found in $23.8 \%, 33.3 \%$ and $31.0 \%$ of patients, respectively. Anti- $\beta 2$-glycoprotein I and lupus anticoagulant were positive in $4.8 \%$ and $11.9 \%$ of patients, respectively. Factor V Leiden mutation analysis was positive in $6.1 \%$ of patients. Coagulation abnormality did not differ significantly between the patients with and without SVT.

Conclusions SVT is more common in patients with necrotizing pancreatitis, suggesting that local inflammation plays a major role in its causation. Thrombophilia is seen in one third of patients with AP but does not seem to increase the risk of SVT.

Keywords Acute pancreatitis, contrast-enhanced computed tomography, protein C, protein S, pancreatic necrosis, Doppler, inflammation

Ann Gastroenterol 2018; 31 (3): 371-378

\section{Introduction}

Acute pancreatitis (AP) is an acute inflammatory process of the pancreas with wide clinical presentations, ranging from mild discomfort to catastrophic debility and even death $[1,2]$. The inflammatory process may be limited to the pancreas or may involve regional or distant organ systems,

Departments of ${ }^{\mathrm{a}}$ Gastroenterology (Sobur Uddin Ahmed, Surinder S. Rana, Ravi Sharma); ${ }^{b}$ Hematology (Jasmina Ahluwalia, Neelam Varma); 'Surgery

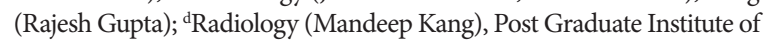
Medical Education and Research (PGIMER), Chandigarh, India

Conflict of Interest: None

Correspondence to: Prof. Surinder Singh Rana, Department of Gastroenterology, PGIMER, Chandigarh, 160012 India, e-mail: drsurinderrana@yahoo.co.in

Received 6 December 2017; accepted 22 January 2018; published online 2 March 2018

DOI: https://doi.org/10.20524/aog.2018.0242 leading to local or systemic complications $[1,2]$. The arterial and venous complications of AP, including thrombosis and pseudoaneurysms, are an important cause of morbidity and mortality $[3,4]$.

Splanchnic venous system thrombosis (SVT) is one of the common vascular complications of AP [5]. There is wide variation in the incidence of SVT in AP between different studies, with reported frequencies ranging from as low as $1.8 \%$ to as high as $36.5 \%$ [6-9]. Several studies have looked at the pathophysiological mechanisms associated with SVT [8-10]. Factors that have been implicated are pancreatic and peripancreatic edema, inflammatory cellular infiltration, direct injury to blood vessels and fluid collection compressing the vessels and causing stasis of blood flow. Peri-pancreatic inflammatory changes or fluid collecstions create a milieu that promotes the development of SVT $[9,11]$.

Thrombophilia has also been implicated as a cause of SVT in AP but this has not been proven conclusively. Deficiencies of natural anticoagulants, such as proteins $\mathrm{C}$ and $\mathrm{S}$, have been found in patients with AP [12] and can lead to SVT [13]. There 
are insufficient data in the literature regarding the role of other coagulation parameters in AP, such as anticardiolipin antibody, lupus anticoagulant, and anti- $\beta 2$ glycoprotein I ( $\beta 2 \mathrm{GPI})$ [14].

Moreover, there is a paucity of literature on the natural history of SVT in AP, with the majority of studies reporting that complications directly related to SVT are infrequent and that most of the patients remain asymptomatic with regard to splanchnic vein thrombosis $[8,9]$. In addition, spontaneous recanalization of a thrombosed splanchnic venous system has been reported in up to $30 \%$ of patients [15].

We conducted this prospective study to evaluate the frequency, risk factors and extent of SVT in patients with AP and its impact on outcome parameters including the presence or absence of necrosis, organ failure, need for intervention, and mortality. These patients also underwent a detailed analysis of coagulation parameters to evaluate the role of thrombophilia in the causation of SVT in AP.

\section{Patients and methods}

\section{Patients}

Eligible patients presenting to our center between January 2015 and June 2016 were included if they had acute pancreatitis as defined by the revised Atlanta criteria [16]. The exclusion criteria were as follows: chronic pancreatitis; past history of acute pancreatitis; known coagulation disorders; taking medications that can alter coagulation parameters, e.g. warfarin and low-molecular-weight heparin; pancreatic or other malignancy; pregnancy; chronic liver disease; disease known to alter coagulation parameters; and unwillingness to provide consent.

\section{Severity assessment}

For patients with acute pancreatitis, the course and severity were assessed in terms of mortality, organ failure (modified Marshall scoring system), complications (necrosis, walled-off pancreatic necrosis, pseudocyst), computed tomography (CT) severity index (CTSI) and modified CTSI (MCTSI), bedside index of severe AP (BISAP) score, and systemic inflammatory response syndrome (SIRS). Any intervention was assessed in terms of indication for intervention, nature of intervention, and time of intervention. Informed consent to participation in the study was received from either the patient or his/her close relative and the study protocol was approved by the institute ethics committee (No. INT/IEC/2015/200).

\section{Data records}

Baseline characteristics, including demographic, history and examination findings, and baseline investigations were recorded. Hematologic data and renal and liver biochemistry parameters were recorded within $24 \mathrm{~h}$ of admission. An abdominal ultrasound examination was performed in all patients within $24 \mathrm{~h}$ of admission. Contrast-enhanced CT (CECT) of the abdomen was performed within 5-7 days from the onset of pain to stage the severity according to the CTSI and MCTSI, and also to determine the presence of SVT. Those patients having SVT on CECT underwent a detailed coagulation profile study including prothrombin time (PT), activated partial thromboplastin time (aPTT), d-dimers, fibrinogen, protein C, protein S, antithrombin (AT) III, lupus anticoagulant, anticardiolipin antibody, $\beta 2$ GPI and factor V Leiden (FVL) mutation analysis. The coagulation profile was also studied in age- and sex-matched AP patients without SVT seen during the same period and this group of patients served as controls.

\section{Coagulation tests}

Citrated samples were collected for measurement of protein $\mathrm{C}$, protein S, AT III, lupus anticoagulant (diluted Russell viper venom test and silica clotting time), d-dimer and coagulation screen (PT, aPTT, fibrinogen). Serum was collected for anticardiolipin and anti- $\beta 2$ GPI antibody assays. An EDTA sample was collected for FVL mutation analysis. The following tests were performed on plasma using a fully automated coagulation analyzer (ACL TOP 500): coagulation screen, protein $\mathrm{C}$ functional activity by chromogenic assay using the manufacturer instructions, free protein-S antigenic assay by latex immunoassay, and antithrombin functional activity by chromogenic assay. D-dimer assay was performed by latex agglutination assay on the ACL TOP 500 coagulation analyser. The anticardiolipin and anti- $\beta 2 \mathrm{GPI}$ assays were performed using commercial ELISA kits (ORGENTEC diagnostic GmbH). FVL mutation was tested by polymerase chain reaction restriction fragment length polymorphism using Mnl I restriction enzymes.

\section{Follow up}

Patients were prospectively followed clinically till recovery or death after admission. A symptom review was carried out at regular intervals and any new findings were recorded. Repeat ultrasound imaging with color Doppler was performed at the discretion of the clinician to inspect the status of fluid/necrotic collections, to determine whether there was progression/ resolution of thrombus in the splanchnic venous system, and to follow the course of the SVT. Any investigation or imaging was repeated at the discretion of the clinician. Patients were given standard medical care throughout the study period. Any signs of organ failure, fever, or infection at any time during the follow-up period were noted.

\section{Outcome}

Outcome was measured in terms of morbidity and mortality, need for intervention, type of intervention, time of 
intervention, indication for the intervention and the length of hospital stay.

\section{Statistical analysis}

Continuous variables were reported as mean, standard deviation and range, while categorical variables were reported as frequencies and percentages. For comparisons between patients with and without SVT, or between normal and abnormal coagulation parameters, continuous variables were compared using an independent $t$-test, and categorical variables using the chi-square test. The significance level was set at $<0.05$.

\section{Results}

One hundred eleven patients were prospectively selected, of whom 15 were excluded (recurrent pancreatitis in 9, coexistent rheumatoid arthritis in 2, coexistent chronic liver diseases in 2, coexistent ulcerative colitis in 1 , and 1 patient where CT could not be done). The remaining 96 patients (76\% male; mean age $31.85 \pm 13.34$ years, range $15-77$ years) were evaluated with CECT of the abdomen for the presence of SVT. The most common etiology of AP was alcohol (45.8\%), followed by gallstone disease $(32.3 \%)$, whereas $13.5 \%$ of patients had idiopathic AP. All the patients had abdominal pain on presentation.

\section{Imaging}

CECT of the abdomen was performed in 96 patients. Eighty patients $(83.3 \%)$ had necrotizing pancreatitis, while $16(16.7 \%)$ had non-necrotizing pancreatitis. A collection was present in $84(87.5 \%)$ patients, of whom $70(83.4 \%)$ had acute necrotic collection and $7(8.3 \%)$ had acute fluid collection. Nine patients underwent post-admission CECT of the abdomen $\geq 28$ days after onset of pain and 7 (8.3\%) of them had walledoff necrosis. The mean CTSI of the patients was $6.88 \pm 2.81$ and the mean MCTSI was $8.29 \pm 2.11$. More than half of the patients
(55.2\%) had CTSI $>6$ and almost three quarters (74\%) had MCTSI $>6$.

\section{Severity}

Severity was assessed using the revised Atlanta classification system (2012), depending on the presence or absence of organ failure and local or systemic complications [16]. Mild, moderate, and severe were present in $5.2 \%, 36.5 \%$, and $58.3 \%$ of the patients, respectively. Organ failure (OF) was determined based on the modified Marshall scoring system and was present in $61(63.5 \%)$ of the 96 patients. Five patients $(5.2 \%)$ had transient OF and 56 (58.3\%) had persistent OF. Forty-eight (50.0\%) patients had single OF, $12(12.5 \%)$ patients had double $\mathrm{OF}$, and one patient had triple OF.

\section{SVT}

SVT was present in 26 (27.1\%) patients. A single vessel, two vessels and all three vessels were involved in 19 (73.1\%), $3(11.5 \%)$, and $4(15.4 \%)$ patients, respectively. The most commonly involved vessel was the splenic vein in $22(84.6 \%)$ patients, followed by the portal vein in $11(42.3 \%)$ patients and the superior mesenteric vein in $4(15.3 \%)$. Table 1 shows the pattern of vascular thrombosis.

\section{Comparison of patients with and without SVT}

When patients with SVT were compared to those without, there was no significant difference between the two groups with regard to age, sex, duration of hospitalization or etiology of AP. However, on clinical presentation, a lump or pleural effusion were present in significantly more patients in the SVT group compared with the no-SVT group (Table 2). Laboratory parameters were comparable between the two groups. When the outcome variables were compared between the two groups, the presence of necrotizing pancreatitis, CTSI $>6$, and MCTSI $>6$ were significantly associated with SVT. There was no significant association of SVT with BISAP $>2$, presence of SIRS, presence and number of organ failure, severity, need for intervention, or mortality (Table 3).

Table 1 Patterns of splanchnic vascular thrombosis (SVT)

SVT $(\mathrm{N}=26)$

\begin{tabular}{|c|c|c|c|c|c|c|c|c|}
\hline \multicolumn{3}{|c|}{ Single vessel } & \multicolumn{3}{|c|}{ Double vessel } & \multicolumn{3}{|c|}{ Triple vessel } \\
\hline Vessel & No. & $\%$ & Vessel & No. & $\%$ & Vessel & No. & $\%$ \\
\hline SplV & 15 & 57.6 & $\mathrm{SplV+PV}$ & 3 & 11.5 & $\mathrm{SplV}+\mathrm{PV}+\mathrm{SMV}$ & 4 & 15.4 \\
\hline PV & 4 & 15.4 & SplV+SMV & 0 & 0 & & & \\
\hline SMV & 0 & 0 & $\mathrm{PV}+\mathrm{SMV}$ & 0 & 0 & & & \\
\hline Total & 19 & 73.1 & & 3 & 11.5 & & 4 & 15.4 \\
\hline
\end{tabular}




\section{Follow up}

None of the recovered patients had variceal bleeding or intestinal ischemia on 2-month to 12 -month follow up. Followup Doppler imaging was performed in 7 of 26 patients with SVT at one year. Thrombosis resolved in 5 patients $(71.4 \%)$ without any specific intervention other than standard care for AP. Anticoagulants were not used in any of the patients. There was no significant difference between those with or without resolution of thrombosis as regards the outcome parameters, including pattern of SVT, presence or absence of necrosis, CTSI/MCTSI, organ failure, severity, need for any intervention, and mortality.

\section{Coagulation workup}

A detailed procoagulation workup, including protein C, protein S, AT III, lupus anticoagulant, anticardiolipin antibody, $\beta 2 \mathrm{GPI}$, PT, aPTT, fibrinogen, and d-dimer assay, was performed in 42 patients, 18 with SVT and 24 without. Samples for coagulation studies were collected within 4 weeks of the onset of pain in 21 patients (early group) and after 4 weeks in 21 patients (late group). FVL mutation analysis was performed in 33 patients, 19 without SVT and 14 with SVT. During the procoagulant workup, at least one abnormality was found in $25(59.5 \%)$ patients. Protein C deficiency was found in 10 patients $(23.8 \%)$, protein $\mathrm{S}$ deficiency in 14 patients (33.3\%), and AT III deficiency in 13 patients (31\%). $\beta 2 \mathrm{GPI}$ was positive in 2 patients $(4.8 \%)$ and lupus anticoagulant was positive in 5 patients (11.9\%). No patient had an anticardiolipin antibody abnormality. Of the 33 patients undergoing FVL mutation analysis, $2(6.1 \%)$ were found to have a heterozygous

Table 2 Comparison of clinical features in patients of acute pancreatitis with or without splanchnic vascular thrombosis (SVT)

\begin{tabular}{lccc}
\multirow{2}{*}{ Symptoms } & \multicolumn{2}{c}{$\mathrm{n}(\%)$} & P-value \\
\cline { 2 - 3 } & No SVT (N=70) & SVT (N=26) \\
\hline Abdominal pain & $70(100.0)$ & $26(100.0)$ & 1.0 \\
Vomiting & $35(50.0)$ & $12(46.2)$ & 0.738 \\
Fever & $23(32.9)$ & $14(53.8)$ & 0.060 \\
$\begin{array}{lccc}\text { Abdominal } \\
\text { distension }\end{array}$ & $51(72.9)$ & $19(73.1)$ & 0.983 \\
Oliguria & $17(24.3)$ & $4(15.4)$ & 0.348 \\
$\begin{array}{l}\text { Shortness of } \\
\text { breath }\end{array}$ & $34(48.6)$ & $11(42.3)$ & 0.585 \\
Jaundice & $10(14.3)$ & $3(11.5)$ & 0.727 \\
\hline $\begin{array}{l}\text { Pedal edema } \\
\text { Lump in } \\
\text { abdomen }\end{array}$ & $4(5.7)$ & $2(7.7)$ & 0.722 \\
\hline $\begin{array}{l}\text { Ascites } \\
\text { Pleural effusion }\end{array}$ & $20(28.6)$ & $13(50.0)$ & 0.049 \\
\hline
\end{tabular}

mutation. Among the global coagulation parameters, some abnormality was found in most of the patients. Prolonged PT was found in 41 of the 42 patients $(97.6 \%)$ while prolonged aPTT was found in only 2 patients (4.8\%). Fibrinogen level was elevated in 23 patients $(54.8 \%)$, while d-dimer testing was positive in 36 patients (85.7\%). More patients in the early group (coagulation study within 4 weeks of pain onset) had abnormalities in AT III, fibrinogen, and d-dimer compared to the late group (coagulation study $>4$ weeks after pain onset) (Table 4).

When patients with normal procoagulants were compared to those with an abnormal procoagulant workup, there was no significant difference between the groups with regard to age, sex, duration of hospitalization, etiology or any of the outcome variables (Table 5).

\section{Coagulation parameters and SVT}

Among the 26 patients with SVT, 18 underwent a procoagulant workup, as did 24 patients without SVT. Among the patients with SVT an abnormal result was found in $8(44.4 \%)$ patients, while of the 24 patients without SVT 7 (29.2\%) had abnormal results. The difference between the two groups was not statistically significant (Table 6).

\section{Discussion}

Thrombosis involving the splanchnic veins is a known and common complication of AP. The reported incidence of SVT in previous studies ranged from 1.8-36.5\% [6-9,17-19]. In our cohort of 96 patients, SVT was found in 26 (27.1\%) patients. The most commonly involved vessel was the splenic vein in 22 of $26(84.6 \%)$, followed by the portal vein in $11(42.3 \%)$. The superior mesenteric vein was the least common vessel to be involved, with thrombosis observed in only 4 (15.3\%) patients. This pattern of vascular involvement is consistent with previous studies $[8,9]$. Almost all the previous studies reported the splenic vein to be the most commonly involved vessel.

We did not find any significant correlation between the presence or absence of SVT and patients' age, sex, or the etiology of AP. A study by Easler et al also found no significant correlation between these two groups and age, sex or etiological factors [9]. In contrast, Zhou et al found that male sex was associated with a higher probability of SVT and that an idiopathic cause was more common in patients without SVT. However, on multiple logistic regression analysis these factors did not differ significantly between the two groups [20]. We also found that presence of a palpable abdominal lump was significantly associated with the presence of SVT $(50 \%$ vs. 28.6\%; $\mathrm{P}=0.049$ ). A palpable lump in case of AP is usually due to an inflammatory mass or a collection. The presence of pancreatic or peri-pancreatic collection and inflammation has been postulated as a cause of SVT [4,11]. Pleural effusion was also seen in a significantly greater number of patients with SVT, 
Table 3 Comparison of outcome variable between patients with and without splanchnic vascular thrombosis (SVT)

\begin{tabular}{|c|c|c|c|c|}
\hline \multirow[t]{2}{*}{ Parameters } & \multicolumn{3}{|c|}{ n (\%) } & \multirow[t]{2}{*}{ P-value } \\
\hline & No SVT $(\mathrm{N}=70)$ & SVT $(\mathrm{N}=26)$ & Total $(\mathrm{N}=96)$ & \\
\hline Necrotizing pancreatitis & $55(78.6)$ & $25(96.2)$ & $80(83.3 \%)$ & 0.04 \\
\hline \multicolumn{5}{|l|}{$\%$ of necrosis } \\
\hline$<30 \%$ & $22(40.0)$ & $5(20.0)$ & $27(33.8)$ & \multirow[t]{3}{*}{0.214} \\
\hline $30-50 \%$ & $8(14.5)$ & $5(20.0)$ & $13(16.2)$ & \\
\hline$>50 \%$ & $25(44.5)$ & $15(60.0)$ & $40(50.0)$ & \\
\hline CTSI $>6$ & $33(47.1)$ & $20(76.9)$ & $53(55.2)$ & 0.009 \\
\hline Modified CTSI $>6$ & $47(67.1)$ & $24(92.3)$ & $71(74.0)$ & 0.017 \\
\hline BISAP $>2$ & $22(31.4)$ & $10(38.5)$ & $32(33.3)$ & 0.516 \\
\hline SIRS present & $64(91.4)$ & $22(84.6)$ & $86(89.6)$ & 0.331 \\
\hline Organ failure & $44(62.9)$ & $17(65.4)$ & $61(63.5)$ & 0.819 \\
\hline \multicolumn{5}{|l|}{ Number of failed organs } \\
\hline Single organ & $36(51.4)$ & $12(46.2)$ & $48(50.0)$ & \multirow[t]{3}{*}{0.614} \\
\hline Two organs & $7(10.0)$ & $5(19.2)$ & $12(12.5)$ & \\
\hline Multiple organs & $1(1.4)$ & $0(0.0)$ & $1(1.0)$ & \\
\hline \multicolumn{5}{|l|}{ Atlanta severity } \\
\hline Mild & $4(5.7)$ & $1(3.8)$ & $5(5.2)$ & \multirow[t]{3}{*}{0.894} \\
\hline Moderately severe & $26(37.1)$ & $9(34.6)$ & $35(36.5)$ & \\
\hline Severe & $40(50.7)$ & $16(61.5)$ & $56(58.3)$ & \\
\hline PCD & $25(35.7)$ & $14(53.8)$ & 39 (40.6) & 0.108 \\
\hline Endotherapy & $3(4.3)$ & $1(3.8)$ & $4(4.2)$ & 0.924 \\
\hline Surgery & $3(4.3)$ & $1(3.8)$ & $4(4.2)$ & 0.924 \\
\hline Mortality & $6(8.6)$ & $2(7.7)$ & $8(8.3)$ & 0.890 \\
\hline
\end{tabular}

CTSI, computed tomography severity index; BISAP bedside index of severe acute pericarditis; SIRS systemic inflammatory response syndrome; PCD, percutaneous catheter drainage

Table 4 Coagulation parameters; early ( $<4$ weeks) vs. late ( $>4$ weeks)

\begin{tabular}{|c|c|c|c|c|c|}
\hline \multirow[t]{2}{*}{ Parameters } & \multicolumn{2}{|c|}{ Early $(\mathrm{N}=21)$} & \multicolumn{2}{|c|}{ Late $(\mathrm{N}=21)$} & \multirow[t]{2}{*}{$\mathrm{P}$-value } \\
\hline & $\mathrm{n}$ & $\%$ & $\mathrm{n}$ & $\%$ & \\
\hline Protein C & 6 & 28.57 & 4 & 19.04 & 0.469 \\
\hline Protein S & 7 & 33.33 & 7 & 33.3 & 10.0 \\
\hline $\begin{array}{l}\text { Antithrombin } \\
\text { III }\end{array}$ & 10 & 47.61 & 3 & 14.28 & 0.019 \\
\hline $\begin{array}{l}\text { Lupus } \\
\text { anticoagulant }\end{array}$ & 2 & 9.52 & 3 & 14.28 & 0.634 \\
\hline $\begin{array}{l}\text { Anticardiolipin } \\
\text { antibody }\end{array}$ & 0 & - & 0 & - & - \\
\hline$\beta 2 \mathrm{GPI}$ & 1 & 4.76 & 1 & 4.76 & 10.0 \\
\hline PT & 21 & 100 & 20 & 95.2 & 0.311 \\
\hline aPTT & 1 & 4.76 & 1 & 4.76 & 10.0 \\
\hline Fibrinogen & 15 & 71.42 & 8 & 38.09 & 0.03 \\
\hline d-dimer & 21 & 100 & 15 & 71.42 & 0.008 \\
\hline
\end{tabular}

which may be related to a higher degree of local inflammation in AP patients with pleural effusion.

Pancreatic and peri-pancreatic necrosis and the associated inflammatory process cause vascular inflammation and infiltration of vessels by inflammatory cells, inducing stasis leading to thrombus formation $[8,9]$. In our study, consistently with this hypothesis, the presence of necrotizing pancreatitis was significantly associated with a greater probability of developing SVT. The percentage of necrosis was higher in patients with SVT compared to those without. Previous studies have reported that a higher CTSI is associated with a greater probability of SVT $[18,20]$. In our study, severe pancreatitis, defined by a CTSI $>6$, was seen in a significantly greater number of patients with SVT compared to those without SVT (76.9\% vs. $47.1 \%, \mathrm{P}=0.009)$.

We found no significant association between the need for any intervention, such as surgery or endotherapy, in patients with SVT compared to those without. Though the need for percutaneous catheter drainage was more common in patients with SVT compared to those without SVT, the difference did not reach the statistical significance. Earlier studies have reported that patients with SVT have longer hospital stays compared to those without [6-9,17-19]. Mortality as a direct 
Table 5 Comparison of outcome variables between patients with normal and abnormal procoagulant results

\begin{tabular}{|c|c|c|c|c|}
\hline \multirow[t]{2}{*}{ Parameters } & \multicolumn{3}{|c|}{ n (\%) } & \multirow[t]{2}{*}{ P-value } \\
\hline & Normal Procoagulant $(\mathrm{N}=17)$ & Abnormal Procoagulant $(\mathrm{N}=25)$ & Total $(\mathrm{N}=42)$ & \\
\hline Necrotizing pancreatitis & $16(94.1)$ & $22(88.0)$ & $38(90.5$ & 0.507 \\
\hline \multicolumn{5}{|l|}{$\%$ of necrosis } \\
\hline$<30 \%$ & $4(25.0)$ & $3(13.6)$ & $7(18.4)$ & \multirow[t]{3}{*}{0.222} \\
\hline $30-50 \%$ & $1(6.2)$ & $6(27.3)$ & $7(18.4)$ & \\
\hline$>50 \%$ & $11(68.8)$ & $13(59.1)$ & $24(63.2)$ & \\
\hline CTSI $>6$ & $12(70.6)$ & $18(72.0)$ & $30(71.4)$ & 0.921 \\
\hline Modified CTSI $>6$ & $12(70.6)$ & $18(72.0)$ & $30(71.4)$ & 0.078 \\
\hline BISAP $>2$ & $4(23.5)$ & $10(40.0)$ & $14(33.3)$ & 0.266 \\
\hline SIRS present & $14(82.4)$ & $22(88.0)$ & $36(85.7)$ & 0.608 \\
\hline Organ failure & $10(58.8)$ & $17(68.0)$ & $27(64.3)$ & 0.542 \\
\hline \multicolumn{5}{|l|}{ Number of failled organs } \\
\hline Single organ & $8(47.1)$ & $13(52.0)$ & $21(50.0)$ & \multirow[t]{3}{*}{0.813} \\
\hline Two organs & $2(11.8)$ & $4(16)$ & $6(14.3)$ & \\
\hline Multiple organs & - & - & - & \\
\hline \multicolumn{5}{|l|}{ Atlanta severity } \\
\hline Mild & $1(5.9)$ & $0(0.0)$ & $1(2.4)$ & \multirow[t]{3}{*}{0.458} \\
\hline Moderately severe & $7(41.2)$ & $10(40.0)$ & $17(40.5)$ & \\
\hline Severe & $9(52.9)$ & $15(60.0)$ & $24(57.1)$ & \\
\hline PCD & $7(41.2)$ & $9(36.0)$ & $16(38.1)$ & 0.735 \\
\hline Endotherapy & $1(5.9)$ & $0 .(0.0)$ & $1(2.4)$ & 0.220 \\
\hline Surgery & $0(0.0)$ & $1(4.0)$ & $1(2.4)$ & 0.404 \\
\hline Mortality & $2(11.8)$ & $1(4.0)$ & $3(7.1)$ & 0.556 \\
\hline
\end{tabular}

CTSI, computed tomography severity index; BISAP bedside index of severe acute pericarditis; SIRS systemic inflammatory response syndrome; PCD, percutaneous catheter drainage

Table 6 Coagulation parameters in patients with acute pancreatitis with or without splanchnic vascular thrombosis (SVT)

\begin{tabular}{|c|c|c|c|c|}
\hline \multirow[t]{2}{*}{ Coagulation parameters } & \multicolumn{3}{|c|}{$\mathrm{n}(\%)$} & \multirow[t]{2}{*}{ P-value } \\
\hline & No SVT $(\mathrm{N}=24)$ & SVT $(\mathrm{N}=18)$ & Total $(\mathrm{N}=42)$ & \\
\hline Abnormal procoagulant & $17(70.8)$ & $8(44.4)$ & $25(59.5)$ & 0.085 \\
\hline Protein C deficiency & $6(25.0)$ & $4(22.2)$ & $10(23.8)$ & 0.834 \\
\hline Protein S deficiency & $7(29.2)$ & $7(38.9)$ & $14(33.3)$ & 0.508 \\
\hline Antithrombin III deficiency & $9(37.5)$ & $4(22.2)$ & $13(31.0)$ & 0.289 \\
\hline Lupus anticoagulant positive & $4(16.7)$ & $1(5.6)$ & $5(11.9)$ & 0.271 \\
\hline Anticardiolipin antibody positive & $0(0.0)$ & $0(0.0)$ & $0(0.0)$ & - \\
\hline$\beta 2$ GPI positive & $1(4.2)$ & $1(5.6)$ & $2(4.8)$ & 0.834 \\
\hline FVL mutation analysis & $\mathrm{N}=19$ & $\mathrm{~N}=14$ & $\mathrm{~N}=33$ & \\
\hline FVL mutation positive & $2(6.1)$ & $0(0.0 \%)$ & $2(6.1 \%)$ & 0.210 \\
\hline
\end{tabular}

32 GPI, anti- $\beta 2$ glycoprotein I; FVL, factor V Leiden

consequence of SVT has not been reported in patients with AP, although SVT has a high mortality outside the setting of AP $[21,22]$. We also found no significant difference in mortality between the two groups.
Ours is a large study as far as the coagulation workup in AP is concerned. Among the procoagulant workup, at least one abnormality was present in more than half of the patients. Previous studies have reported that systemic activation of 
the coagulation system commonly occurs in over half of the critically ill patients with severe AP [12]. Proteins C and S, and AT III deficiencies were found in $23.8 \%, 33.3 \%$, and $31.0 \%$ of patients respectively, similar to previous studies [12,23-25]. Deficiency of these natural anticoagulants occurs as part of the systemic inflammatory response. Systemic inflammation causes downregulation of hepatic synthetic functions, leading to a deficiency of these proteins [12]. The high number of patients with such a deficiency in our study can be explained by the large number of patients who had SIRS. Ours is the largest study looking into lupus anticoagulant, $\beta 2 \mathrm{GPI}$, anticardiolipin antibody, and FVL mutation analysis. These procoagulant parameters have previously been evaluated in only a small number of AP patients with SVT [7].

We found no significant correlation between patients having normal or abnormal procoagulant and any of the outcome variables. This is in contrast to previous studies, where higher mortality and multiple organ failure were found to be associated with abnormal procoagulation parameters [12,26]. We found prolongation of PT in most (97.6\%) of the patients and prolonged aPTT in only few (4.8\%) patients, consistent with previous studies [27,28]. Prolongation of PT may be secondary to disseminated intravascular coagulation (DIC), associated with severity inflammatory processes such as pancreatitis; or it may be secondary to impaired hepatic synthetic function caused by cytokine as a part of SIRS. In DIC, the level of fibrinogen is decreased and that of d-dimer is elevated. This is in contrast to our study, as we found increased fibrinogen levels in $54.8 \%$ of patients. However, an increased fibrinogen level can be due to the fact that fibrinogen is an acute phase reactant, whose level may be increased in acute inflammatory conditions like AP. Consistently with this, in our study we found that high fibrinogen levels were found in a significantly higher number of patients whose coagulation study was performed early in the course of AP ( $<4$ weeks) compared to those whose study was performed later ( $>4$ weeks). The increased level of d-dimer (in $87.5 \%$ of patients) indicates fibrinolysis, suggesting that fibrinogen production and fibrinolysis go hand in hand in AP. This is consistent with our finding that a significantly higher number of patients had increased d-dimer levels when their coagulation study was done early rather than late in the course of AP.

Compared to the patients without SVT, patients with SVT did not show any significant difference in the results of their coagulation studies. In fact, an overall abnormality of procoagulant parameters was seen more commonly in the group without SVT. This was probably due to a systemic inflammatory response and cytokine production in AP, causing impaired hepatic synthetic function (protein C and S deficiency) or consumption coagulopathy secondary to diffuse microvascular thrombi. One of our patients with positive lupus anticoagulant had involvement of all three vessels in thrombosis. Even if we assume that the presence of positive lupus anticoagulant caused this extensive thrombosis, two other patients in whom all three vessels were involved had no abnormality in procoagulant parameters, indicating that extent of vascular thrombosis does not have any correlation with normal or abnormal coagulation parameters.
Follow-up ultrasound Doppler imaging showed that SVT resolved in most of the patients within 1 year without the use of any anticoagulation therapy. Studies by Gonzalez et al and Harris et al reported that one third of patients with SVT had resolution of thrombus without anticoagulants $[7,8]$. However, some authors have reported that anticoagulant treatment is useful and should be applied in patients with SVT [29]. Complications in AP, particularly those related to SVT, are infrequent. We found no complication specifically related to SVT, such as variceal bleeding or intestinal ischemia.

Our study had certain important limitations. There was a higher proportion of patients with acute necrotizing pancreatitis in our study; this is because our center is a tertiary care referral institution in North India where a majority of sick cases are referred, whereas patients with mild pancreatitis are usually treated at peripheral centers. In addition, the study population was not homogeneous-being mainly male (76\%) and with alcohol-induced pancreatitis (45.8\%), just a minority of cases having idiopathic pancreatitis-which could have led to bias [20]. Although none of the patients with alcoholinduced pancreatitis had overt liver disease, subclinical chronic liver disease could have influenced their real coagulation status. Other limitations are that few patients with SVT were followed up (7/26 patients) and for shorter durations, while the follow up used Doppler imaging, which always remains an operatordependent method. Finally, there are no established guidelines supporting the use of Doppler imaging at regular intervals

\section{Summary Box}

\section{What is already known:}

- Splanchnic venous system thrombosis (SVT) is one of the common vascular complications of acute pancreatitis (AP)

- Thrombophilia has also been implicated as a cause of SVT in AP but this has not been conclusively proven

- Pancreatic and peri-pancreatic inflammatory processes cause vascular inflammation and infiltration of vessels by inflammatory cells and could also contribute to SVT

\section{What the new findings are:}

- The presence of necrotizing pancreatitis is significantly associated with a higher chance of developing SVT

- The presence of SVT does not significantly impact the outcome of AP

- SVT is not related to normal or abnormal blood coagulation parameters

- In most patients with SVT the thrombosis resolves without any intervention 
for assessing the status of SVT and our selection of a cutoff of 4 weeks after the onset of pain for assessing coagulation parameters was arbitrary.

In conclusion, SVT is a common finding in AP and is significantly associated with the presence of necrotizing pancreatitis. It does not significantly impact the outcome of the disease, it is not related to normal or abnormal blood coagulation parameters, and in most patients it resolves without any intervention.

\section{References}

1. Beger HG. Surgical management of necrotizing pancreatitis. Surg Clin North Am 1989;69:529-549.

2. Neoptolemos JP, Raraty M, Finch M, Sutton R. Acute pancreatitis: the substantial human and financial costs. Gut 1998;42:886-891.

3. Gaa J, Georgi M. Non-invasive imaging of abdominal vascular pathologies. Eur Radiol 1998;8:507-516.

4. Mallick IH, Winslet MC. Vascular complications of pancreatitis. JOP 2004;5:328-337.

5. Barge JU, Lopera JE. Vascular complications of pancreatitis: role of interventional therapy. Korean J Radiol 2012;13 Suppl 1:S45-S55.

6. Dörffel T, Wruck T, Rückert RI, Romaniuk P, Dörffel Q, Wermke W. Vascular complications in acute pancreatitis assessed by color duplex ultrasonography. Pancreas 2000;21:126-133.

7. Harris S, Nadkarni NA, Naina HV, Vege SS. Splanchnic vein thrombosis in acute pancreatitis: a single-center experience. Pancreas 2013;42:1251-1254.

8. Gonzelez HJ, Sahay SJ, Samadi B, Davidson BR, Rahman SH. Splanchnic vein thrombosis in severe acute pancreatitis: a 2 years single-institution experience. HPB (Oxford) 2011;13:860-864.

9. Easler J, Muddana V, Furlan A, et al. Portosplenomesenteric venous thrombosis in patients with acute pancreatitis is associated with pancreatic necrosis and usually has a benign course. Clin Gastroenterol Hepatol 2014;12:854-862.

10. Nadkarni NA, Khanna S, Vege SS. Splanchnic venous thrombosis and pancreatitis. Pancreas 2013;42:924-931.

11. Mendelson RM, Anderson J, Marshall M, Ramsay D. Vascular complications of pancreatitis. ANZ J Surg 2005;75:1073-1079.

12. Badhal SS, Sharma S, Saraya A, Mukhopadhyay AK. Prognostic significance of $\mathrm{D}$-dimer, natural anticoagulants and routine coagulation parameters in acute pancreatitis. Trop Gastroenterol 2012;33:193-199.

13. Lucendo Villarín AJ, Carrión Alonso G, Martín Chavarri S, Allona Kraue M, Prado Rodríguez JR. Acute pancreatitis and protein C deficiency as a cause of mesenteric venous thrombosis. Rev Esp Enferm Dig 2006;98:553-554.
14. Chang KY, Kuo YC, Chiu CT, et al. Anti-cardiolipin antibody associated with acute hemorrhagic pancreatitis. Pancreas 1993;8:654-657.

15. Butler JR, Eckert GJ, Zyromyski NJ, Leonardi MJ, Lillemoe KD, Howard TJ. Natural history of pancreatitis-induced splenic vein thrombosis: a systematic review and meta-analysis of its incidence and rate of gastrointestinal bleeding. HPB (Oxford) 2011;13:839845.

16. Banks PA, Bollen TL, Dervenis C, et al; Acute Pancreatitis Classification Working Group. Classification of acute pancreatitis-2012: revision of the Atlanta classification and definitions by international consensus. Gut 2013;62:102-111.

17. Popa D, Grigorescu B, Hoffner S, Copotoiu C. Vascular complications-poor prognostic factors in severe acute pancreatitis. Acta Medica Marisiensis 2011;57:74-76.

18. Mortelé KJ, Mergo PJ, Taylor HM, et al. Peripancreatic vascular abnormalities complicating acute pancreatitis: contrast-enhanced helical CT findings. Eur J Radiol 2004;52:67-72.

19. Thatipelli MR, McBane RD, Hodge DO, Wysokinski WE. Survival and recurrence in patients with splanchnic vein thromboses. Clin Gastroenterol Hepatol 2010;8:200-205.

20. Zhou J, Ke L, Tong Z, et al. Risk factors and outcome of splanchnic venous thrombosis in patients with necrotizing acute pancreatitis. Thromb Res 2015;135:68-72.

21. Boley SJ, Kaleya RN, Brandt LJ. Mesenteric venous thrombosis. Surg Clin North Am 1992;72:183-201.

22. Kaleya RN, Boley SJ. Acute mesenteric ischemia. Crit Care Clin 1995;11:479-512.

23. Radenković D, Bajec D, Karamarkovic A, et al. Disorders of hemostasis during the surgical management of severe necrotizing pancreatitis. Pancreas 2004;29:152-156.

24. Lindstrom O, Kylanpaa L, Mentula P, et al. Upregulated but insufficient generation of activated protein $\mathrm{C}$ is associated with development of multiorgan failure in severe acute pancreatitis. Crit Care 2006;10:R16.

25. Pettilä V, Kyhälä L, Kylänpää ML, et al. APCAP—activated protein $\mathrm{C}$ in acute pancreatitis: a double-blind randomized human pilot trial. Crit Care 2010;14:R139.

26. Maeda K, Hirota M, Ichihara A, et al. Applicability of disseminated intravascular coagulation parameters in the assessment of the severity of acute pancreatitis. Pancreas 2006;32:87-92.

27. Radenkovic D, Bajec D, Ivancevic N, et al. D-dimer in acute pancreatitis: a new approach for an early assessment of organ failure. Pancreas 2009;38:655-660.

28. Gould T, Mai S, Liaw P. Coagulation abnormalities in acute pancreatitis. In: Rodrigo L (ed). Pancreatitis - treatment and complications. In Tech Europe Rijeka, Croatia 2012, pp. 59-72.

29. Park WS, Kim H, Jeon BJ, Kim SH, Lee SO. Should anticoagulants be administered for portal vein thrombosis associated with acute pancreatitis? World J Gastroenterol 2012;18:6168-6171. 